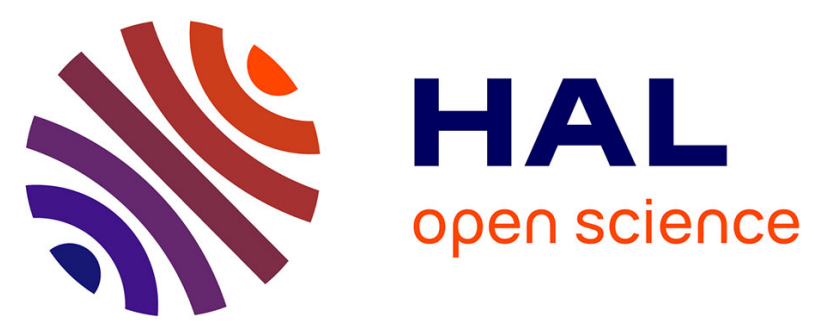

\title{
Impact of divertor configuration on recycling neutral fluxes for ITER-like wall H-mode plasmas
}

E de La Cal, U Losada, A Martín de Aguilera, A Shaw, E Solano, D Alegre, I Balboa, P Carvalho, J Gaspar, I Borodkina, et al.

\section{- To cite this version:}

E de La Cal, U Losada, A Martín de Aguilera, A Shaw, E Solano, et al.. Impact of divertor configuration on recycling neutral fluxes for ITER-like wall H-mode plasmas. Plasma Physics and Controlled Fusion, 2020, 62, pp.035006. 10.1088/1361-6587/ab5fb1 . hal-03150405

\section{HAL Id: hal-03150405 https://hal-amu.archives-ouvertes.fr/hal-03150405}

Submitted on 23 Feb 2021

HAL is a multi-disciplinary open access archive for the deposit and dissemination of scientific research documents, whether they are published or not. The documents may come from teaching and research institutions in France or abroad, or from public or private research centers.
L'archive ouverte pluridisciplinaire HAL, est destinée au dépôt et à la diffusion de documents scientifiques de niveau recherche, publiés ou non, émanant des établissements d'enseignement et de recherche français ou étrangers, des laboratoires publics ou privés. 


\title{
recycling neutral fluxes for ITER-like wall $\mathrm{H}$ -
} 3 mode plasmas

\author{
E. de la Cal ${ }^{1}$, U. Losada ${ }^{1}$, A. Martín de Aguilera ${ }^{1}$, E. Solano ${ }^{1}$, D. Alegre ${ }^{1,2}$, I. Balboa ${ }^{3}$, P. Carvalho ${ }^{3}$, \\ J. Gaspar ${ }^{5}$, A. Shaw ${ }^{3}$, I. Borodkina ${ }^{4}$, S. Brezinsek ${ }^{4}$, D. Douai ${ }^{6}$, C. Giroud ${ }^{3}$, C. Guillemaut ${ }^{3}$, C. \\ Hidalgo $^{1}$, A. Huber ${ }^{3}$, E. Joffrin ${ }^{6}$, T. Loarer ${ }^{6}$, E. de la Luna ${ }^{1}$, A. Manzanares ${ }^{1}$, F. Militello ${ }^{3}$, L. de \\ Pablos $^{1}$, S. Wiesen ${ }^{4}$ and JET contributors ${ }^{*}$ \\ ${ }^{1}$ Laboratorio Nacional de Fusión, CIEMAT, Av. Complutense 40, E-28040 Madrid, Spain \\ ${ }^{2}$ Departamento de Ingeniería Energética, UNED, C/Juan del Rosal 12, E-28040 Madrid, Spain \\ ${ }^{3}$ CCFE Fusion Assoc., Culham Science Centre, Abingdon, OX14 3DB, UK \\ ${ }^{4}$ Forschungszentrum Jülich, Institut für Energie- und Klimaforschung- und Plasmaphysik, 52425, Jülich, Germany \\ ${ }^{5}$ Aix Marseille Univ, CNRS, IUSTI, Marseille, France \\ ${ }^{6}$ CEA, IRFM, F-13108 Saint-Paul-lez-Durance, France \\ "See the author list of "X. Litaudon et al 2017 Nucl. Fusion 57 102001" \\ E-mail: e.delacal@ciemat.es
}

\section{Abstract}

It is well known since the last years that in JET, with the ITER-like wall, the performance of high-power Hmode plasmas strongly depends on the divertor magnetic topology. This is generally attributed to the effect of the magnetic field shaping on the neutral flux transport and pumping, which determine in high density H-mode plasmas the pedestal properties and finally the global confinement. In the present work we have analysed for different magnetic configurations the spatial distribution and the dynamic behaviour of the $\mathrm{D}_{\alpha^{-}}$ emission. Experimental observations indicate that for certain configurations, the surface temperature and the $\mathrm{D}_{\alpha}$-emission anomalously increase on top of the inner divertor, which points to thermal outgassing there. This is the region where most Beryllium co-deposits accumulate and most Deuterium becomes trapped. The overheating at this region far from the strike point (SP) is observed to happen in magnetic configurations with reduced distance between the divertor material surface and the Separatrix (Clearance). The neutral flux that appears at the upper inner divertor during a few milliseconds after the ELM-crash, is by more than an order of magnitude larger than the puffing rate and dominates over the rest of the divertor recycling . 
Finally, a preliminary study describes how this thermal fuel outgassing from the co-deposited layers could be intentionally used as a Wall-conditioning technique with plasmas that focalise their particle and heat flux there. This could be used as a Wall isotope exchange technique or for Tritium recovery from regions where Be co-deposits accumulate in JET with the ITER-like wall.

Keywords: Plasma-wall, Recycling, Divertor Configuration, Fuel Trapping, Outgassing, Neutrals

\section{Introduction}

Since the installation of the ITER-like wall (ILW), JET has demonstrated successfully the compatibility of an all-metallic wall with reactor relevant high power H-mode scenarios. The benefit is a reduced fuel trapping in the vacuum vessel by at least one order of magnitude with respect to the Carbon divertor [1-4]. The latter exhibited a prohibitively large wall fuel accumulation due to the strong chemical reactivity of Carbon with Hydrogen isotopes. The Hydrocarbons generated by the plasma demonstrated to have a high sticking capacity at remote and hidden surfaces producing thick hydrogenated Carbon co-deposits. No reliable cleaning method to remove these deposits was found. The reactor vessel Tritium (T) inventory predicted due to this effect showed that operation was impossible to be maintained under safety limits for a reasonable fusion plasma burning operation time: a few hundred 400 seconds D-T ITER plasmas would have been sufficient to overpass this limit. With such pessimistic predictions, the use of Carbon as plasma facing component for next step burning reactors had to be abandoned and metallic plasma facing components appeared as the alternative. The promising JET-ILW results made the fusion community gain confidence in reactor relevant plasma scenarios with an all-metallic environment and ITER will operate since the beginning with a Beryllium-Tungsten First wall. The originally planed preliminary operation phase with a Carbon divertor has been cut out, thereby reducing costs and time till D-T operation.

Moreover, during this last JET period, valuable experience has been gained in plasma operation with an all-metallic wall, which has been shown to have particularities not faced before with the Carbon phase. New "hidden parameters" appear that are being studied in order to understand the physics of H-mode plasmas [see e.g. 3-4]. For example, during the first years of JET-ILW operation, H-mode confinement was sensibly reduced by at least $20 \%$ in the so-called ITER Baseline scenario (normalized pressure ratio $\beta_{\mathrm{N}} \approx$ 1.5) with respect to the Carbon phase. Only with Nitrogen seeding, good confinement could partially be recovered [5]. This degradation was attributed to the large fuel puffing amounts necessary to reduce the plasma edge temperature at the divertor targets to minimise Tungsten sputtering and its subsequent accumulation in the plasma centre. This is believed to be the origin of the pedestal and central plasma temperature decrease and the performance degradation. More recently it was discovered that global confinement could be improved for specific magnetic configurations, with the best performance (confinement factor $\mathrm{H}_{98 \mathrm{Y}} \approx 1$ ) obtained with the SPs located at the divertor corners near the pumping conducts (here called Corner- or C-configuration), where recycled neutral exhaust is most efficient [6-11, see figure 1].

This observation addresses once again to the role of the neutral density (or pressure) on the scrape-offlayer (SOL) plasma. Generally speaking, high neutral densities degrade the pedestal properties and finally 
the global plasma confinement. Unfortunately, up to date, there is no clear understanding of the physics behind it. Simulations show that energy losses of neutrals entering the confined region are minimised with the SP near the pumping openings at the divertor corners, but the quantitative values don't explain by far the observed differences in confinement [9]. On the other hand, the increase of the main chamber neutral pressure has been linked with the outer SP position distance to the divertor pumping openings at the corner, which affects negatively plasma pedestal pressure [11]. This empirical observation was already disclosed many years ago and the energy confinement time was directly scaled with neutral pressure in the main chamber [see e.g. 12, 13]. A possible explanation of this effect disclosed in [11] links the main chamber neutrals with the radial electric field change that increases the rotational shear at the plasma edge, which finally reduces perpendicular transport. For further bibliography concerning the effect of divertor geometry and magnetic configuration on many different aspects such as the pumping efficiency, target power loads, ionization sources and detachment, etc., the reader is referred to [14]. For modelling aspects of neutrals including molecular dynamics in ITER and its influence on divertor performance the reader is referred to [15] and references therein.

On the other hand, since operation with the ITER-like wall, the physics of Recycling has considerably varied. This was something expected due to the very different characteristics of Carbon (C) and Tungsten (W) when exposed to Deuterium (D) fluxes [1, 16-23]. For example $\mathrm{W}$ has a much larger reflection coefficient compared to $\mathrm{C}$, and a much lower capacity to adsorb D, except in regions where Beryllium (Be) co-deposits grow. Moreover, Recycling, which is the re-emission by the plasma-facing material of D as response of the ion and heat fluxes, should be seen as a dynamic process if transient events as ELMs are present. Therefore, the physics of ELMy scenarios in the SOL and Edge plasma is a complex kinetic plasma-wall interaction process where the neutral, ion and heat transport must be balanced. Some aspects of the Recycling and pedestal recovery dynamics in JET with the new ITER-like wall have been described recently [see e.g. 19-23]. More generic modelling of the dynamics of the surface response during ELMs can be found in [24-25]. There is also an experimental study on the Recycling coefficient fast variations during ELM-like events in the TJ-II stellarator [26]. Finally, a theoretical study of the fuel absorption and reemission kinetics has been done for $\mathrm{W}$ when exposed to ELMs using a Diffusion-Trapping Model, simulating quantitatively the incident power and particle fluxes [27].

In the present work we study the spatial distribution and the dynamic behaviour of the recycling neutral fluxes at the divertor for high-power $\mathrm{H}$-mode plasmas with different magnetic configurations. The total heating power P > 15 MW was mainly through Neutral Beam Injection (NBI) with sometimes a small fraction of Ion Cyclotron Range of Frequencies (ICRF) heating. More specifically the effect of the proximity of the SPs to the divertor corners near the pumping conducts is compared for similar plasmas as done in previous studies [6-11]. The main analysis has been done comparing the spatial distribution of the $\mathrm{D}_{\alpha}$-emission and its dynamics during ELMs at the inner divertor, since the neutral fluxes dominate there for the here studied plasmas.

In a previous work it was already observed [22], that for certain magnetic topologies, the D neutral fluxes strongly increase for high power H-mode plasmas on top of the inner divertor just after the ELMcrash. This was initially surprising because this region is relatively far from the SP position. The D emission came from the region where surface analysis has shown that most D becomes trapped within the 
Be co-deposits that preferentially accumulate there. Therefore we proposed already in [22] that local fuel outgassing due to surface over-heating could be the reason of the increased neutral flux. Also in a recent study, during the change-over from a Deuterium to a Hydrogen campaign, a strong spatial inhomogeneity of the plasma isotope ratio in the divertor was observed [28]. This phenomenon was attributed to local reemission of the fuel isotope of the previous campaign that was trapped in the Be co-deposits of the inner divertor.

The goal of the present work is to try to understand this outgassing phenomenon in more detail. Therefore, we analyse the surface temperature evolution using thermography to show that there is a clear correlation with the $\mathrm{D}_{\alpha}$-emission increase at this divertor region. The neutral flux generated during a few milliseconds after the ELM-crash is estimated using the absolutely calibrated $\mathrm{D}_{\alpha}$-radiance. It is by more than an order of magnitude larger than the puffing rate and dominates the recycling neutral fluxes in the divertor. Different examples indicate that the anomalous overheating of the upper inner divertor tiles that induce the D outgassing is linked to the magnetic configuration and more specifically, to the distance of the plasma Separatrix to the material surfaces.

In a final section it is shown, how this local outgassing from the upper inner divertor region induced by the plasma heating could be used to intentionally desorb the accumulated fuel desorption there. This could be useful for wall isotope control and fuel inventory reduction in future JET campaigns with T [29].

\section{Experimental}

The here analysed discharges are high-power $(\mathrm{P} \geq 15 \mathrm{MW}) \mathrm{H}$-mode plasmas with plasma current $\mathrm{I}_{\mathrm{P}} \geq$ 1.8 MA and a toroidal magnetic field $\mathrm{B}_{\mathrm{T}} \geq 2 \mathrm{~T}$. We compare always plasma pairs with similar heating power, triangularity and gas injection rate but with different divertor magnetic configuration. More specifically the effect of the proximity of the SPs at the divertor corners near the pumping conducts is compared for similar plasmas as in [6-11].

We analyse the visible plasma emission, mainly the $\mathrm{D}_{\alpha}$-atomic line, to get information of the neutral fluxes. The main diagnostic used is a $D_{\alpha}$-spectroscopy system with narrowband-pass filters that views the divertor with 20 chords from an upper port. The radiance is absolutely calibrated and has a temporal resolution of $100 \mathrm{kHz}$. Also, two spectrometers were used two analyse the $\mathrm{D}_{\gamma} / \mathrm{D}_{\alpha}$ ratio. Additionally, we also analyse the visible emission with a Fast camera with up to $70 \mathrm{kHz}$ sampling rate [30] and a slow (30 $\mathrm{Hz}$ ) intensified $\mathrm{D}_{\alpha^{-}}$and $\mathrm{D}_{\gamma^{-}}$filtered camera. Both have a tangential view of the divertor [31].

To measure the divertor surface temperature the thermal radiation is analysed with a Mid Wave Infrared (MWIR) camera and a Near Infrared Range (NIR) filtered visible camera. The MWIR camera [32, 33] works in the 3-5 $\mu \mathrm{m}$ range and has a sampling rate of about $15 \mathrm{~Hz}$. The exposure times for the here analysed plasmas is of about 0.5 milliseconds. The NIR camera is part of the JET Protection System [34, 35]. The camera filters light from the NIR range to extract from the thermal (Blackbody) radiation the surface temperature. The filter is centred at $\lambda=1016 \mathrm{~nm}$ and has a bandwidth of $+/-40 \mathrm{~nm}$. It is absolutely calibrated for $\mathrm{T}>800{ }^{0} \mathrm{C}$ and its recording speed is $50 \mathrm{~Hz}$. Unfortunately, other plasma emission sources can contaminate the thermal radiation from Volume plasma emission such as Bremsstrahlung and Free- 
bound transitions from recombining processes [33]. It should be clarified that the surface emissivity is a free parameter when obtaining the absolute temperature from the thermal emission and depends on the surface properties. Since plasma exposed surfaces can continuously vary their emissivity, a certain uncertainty in the measurement must be kept in mind. Here an emissivity of $0.18-0.24$ (depending on the temperature range) for the upper inner W-coated carbon divertor tiles was selected. The software framework JUVIL [36] was used to analyse thermal and visble camera videos.

\section{Experimental observations at the inner divertor: "Corner" versus "Vertical" configuration}

\section{1 Global plasma modifications}

Figure 1 shows the two divertor magnetic configurations used in discharge \#86533. The plasma had following parameters: $\mathrm{B}_{\mathrm{T}}=2.4 \mathrm{~T}, \mathrm{I}_{\mathrm{P}}=2.5 \mathrm{MA}, \mathrm{P}=17 \mathrm{MW}(\mathrm{NBI})+1.5 \mathrm{MW}(\mathrm{ICRH})$ and was already analysed in a previous work [6]. During the H-mode, the plasma starts first with the inner SP near the divertor pumping openings (here called Corner or C-configuration) and is moved after 2 seconds to the vertical target (here called Vertical- or V-configuration). Shown are also some inner $\mathrm{D}_{\alpha}$-chords (cannels 1 , 3 and 5) and uppermost Langmuir probes (LP). Figure 2 shows several time traces where the strong influence of the divertor magnetic configuration on the plasma parameters can be seen. Plotted are the signals during the H-mode phase of the Neutral Beam Injection (NBI) power, the plasma average density $\left\langle\mathrm{n}_{\mathrm{e}}\right\rangle$, the pedestal electron temperature $\mathrm{T}_{\mathrm{e}}$, the $\mathrm{H}_{98 \mathrm{Y}}$ confinement factor and the integrated inner and outer divertor $\mathrm{D}_{\alpha}$-emission radiance $\mathrm{L}_{\mathrm{D} \alpha}$. The magnetic configuration passes from $\mathrm{C}$ - (black arrow) to $\mathrm{V}$ configuration (red arrow) at $\mathrm{t}=9-10 \mathrm{~s}$. The $\mathrm{D}$ puffing rate $\mathrm{Q}_{0}$ was firstly set to $2.5 \times 10^{22} \mathrm{D} \mathrm{s}^{-1}$ and was reduced to $2 \times 10^{22} \mathrm{D} \mathrm{s}^{-1}$ during the $\mathrm{V}$-configuration (it is schematically shown at the upper frame in blue). Note that even with the puffing reduction, $\left\langle\mathrm{n}_{\mathrm{e}}\right\rangle$ increases. The degradation of the confinement when going to V-configuration is clear: $\mathrm{T}_{\mathrm{e}}$ drops significantly and the confinement factor $\mathrm{H}_{98 \mathrm{Y}}$ decreases from 1.05 to 0.85. The central electron temperature falls from about 6 to $4 \mathrm{keV}$ (not shown). The divertor plasma also shows considerable changes. During the C-configuration, the global inter-ELM $\mathrm{D}_{\alpha}$-fluxes are a factor of 3 larger at the inner divertor than at the outer. When going to the $\mathrm{V}$-configuration they increase by a factor of 3 at the outer and by a factor of 10 at the inner divertor, indicating an enhanced neutral D flux there. The $\mathrm{D}_{\alpha}$-emission dynamics during the ELMs and its spatial distribution changes will be shown in more detail in the next section. These general trends of the plasma parameter when changing the divertor configuration from Corner- to Vertical- also occur in the plasmas shown in the past [6-11].

\section{2 $\mathrm{D}_{\alpha}$-emission response to divertor magnetic topology changes}

Figure 3a shows two frames of the divertor taken with the visible Fast camera during the Vconfiguration of the same plasma \#86533: left, before the ELM and right, after the ELM. Below is shown the CAD-view of the camera to the divertor. The coloured lines indicate schematically the different emission regions: in red the outer SP-line, in blue the inner one and in yellow the upper inner divertor 
region. Note that a strong emission-cloud appears at the high-field-side (HFS) just after the ELM. The accurate localisation of these emission regions where done applying the "Cross-correlation technique" between different ROIs (Region of Interest) of the Fast Camera data and the spatially-well defined $\mathrm{D}_{\alpha^{-}}$ chord radiances looking from the upper port to the divertor. The camera had no filter for this discharge but the dominant emission at the divertor is by far $\mathrm{D}_{\alpha}$-atomic line emission. A proof of this is the high crosscorrelation yield with the $\mathrm{D}_{\alpha}$-spectroscopy channels once the correct lines were obtained. Also, videos of similar discharges with and without $\mathrm{D}_{\alpha}$-filter are nearly equal, except a higher intensity level without the filter (because of the transmission losses). The camera was operated with $10 \mu$ s exposure time and $25 \mathrm{kHz}$ recording speed.

Figure $3 \mathrm{~b}$ shows two video-clips that can be reproduced by clicking on the frames, above the one of the Corner- and below of the Vertical-configuration. The clips show two ELM cycles, corresponding to about $50 \mathrm{~ms}$ of plasma time. One of the clips corresponds to a very similar discharge with the same parameters and configuration change as \#86533. This is because for the high this framing speed the buffer camera memory limits the video recording time to about two seconds and therefore two consecutive "twindischarges" were necessary to record the full H-mode period. The movement of the image is due to the ELMs that produce vibrations on the camera supporting structure that is fastened to the Tokamak vessel. Looking to the videos, we see that in the C-configuration, the ELMs are faster and the inter-ELM interELM equilibrium pattern is rapidly recovered. In contrast, in the V-configuration, a strong emission cloud appears at the HFS just after the ELM-crash that dominates in intensity in the whole divertor. This cloud is located at the upper inner divertor region on top of Tiles 0 and 1 as shown in figure $3 a$.

Figure $4 \mathrm{a}$, upper frame, shows for the same discharge the inter-ELM $\mathrm{D}_{\alpha}$-radiance $\mathrm{L}^{\mathrm{D} \alpha}$ during the configuration change as obtained from the innermost spectroscopy channel that looks to the corner between Tiles 0 and 1 (see figure 1). The intensity increases during the configuration change by a factor of ten. Below is a zoom of the blue frame of the upper figure. It can be seen that in-between ELMs the $D_{\alpha^{-}}$ emission continuously increases (yellow arrows), wehich makes the inter-ELM intensity monotonically increase during the configuration change (red arrow).

The ELM $\mathrm{D}_{\alpha}$-emission dynamics during the stationary phases of the $\mathrm{C}$ - $(\mathrm{t}<9 \mathrm{~s})$ and $\mathrm{V}$ - configuration ( $\mathrm{t}$ $>10 \mathrm{~s}$ ) is shown in figure $4 \mathrm{~b}$ for channel numbers 1,3 and 5 (see figure 1). Below the total inner divertor WI radiance is also shown to visualize the ELM-crashes. The ELM frequency is of about $40 \mathrm{~Hz}$ in both phases. In the C-configuration, all $\mathrm{D}_{\alpha}$-channels have positive peaks during the ELM-crash. Also a secondary peak appears for the views that look to the SP. In the V-configuration the emission dynamics completely changes. The innermost two channels that look to the inner upper horizontal divertor (Tiles 0 and 1) show strong emission peaks that last about $5 \mathrm{~ms}$ longer than the ELM-crash. These correspond to the post-ELM emission cloud seen with the Fast camera. Channel number 3, which looks to the inner divertor corner, shows sometimes no clear spike at the ELM-crash, but the secondary peak as in channel numbers 1 and 2 is present. The emission of channel number 5, that looks at inner SP (channel number 5), decreases just at the ELM-crash during about $8 \mathrm{~ms}$ (red arrow). These so-called "negative ELMs" are characteristic of a (partly-) recombining, cool and high-density SOL plasma that develops near the SP. Note also that during these periods the WI flux decreases, probably due to the reduced $\mathrm{T}_{\mathrm{e}}$ that lowers the sputtering at the targets. 
When comparing quantitatively all divertor $\mathrm{D}_{\alpha}$-channels, including the outer ones, we have that in the approximately $5 \mathrm{~ms}$ post-ELM period, the contribution from the innermost 3 channels is dominant in the $\mathrm{V}$ configuration. Since the $\mathrm{D}_{\alpha}$-radiance $\mathrm{L}_{\mathrm{D} \alpha}$ is absolutely calibrated, the post-ELM neutral D flux $\mathrm{F}_{0}$ at the upper inner divertor region can be estimated assuming Ionizing conditions there:

(1) $\mathrm{F}_{0}=4 \pi \mathrm{A}(\mathrm{S} / \mathrm{XB}) \mathrm{L}_{\mathrm{D} \alpha}$,

where the observing area A corresponds to the projection of the divertor disc of about $12 \mathrm{~cm}$ radial extension that is observed by the three innermost chords and the number of ionizations events per photon $\mathrm{S} / \mathrm{XB} \approx 20$ (see e.g. [22]). We can assume Ionizing conditions since recombination does not contribute at least significantly to the neutral fluxes in this region as is shown in the separate Appendix. From equation (1) we obtain a $\mathrm{D}$ neutral flux of about $\mathrm{F}_{0} \approx 5 \times 10^{23} \mathrm{D} \mathrm{s}^{-1}$ during the post-ELM peak coming from the horizontal upper divertor region covered by the first three $\mathrm{D}_{\alpha}$-chords. This is a significant quantity, a factor of 25 higher than the puffing rate $\left(\approx 2 \times 10^{22} \mathrm{D} \mathrm{s}^{-1}\right)$.

\section{3 Correlation of thermal radiation to post-ELM $D_{\alpha}$-emission}

The post-ELM neutral fluxes from the upper inner divertor region described in the paragraph above were observed systematically for a large number of high power H-mode plasmas (see later for further examples). As already disclosed in a previous work [21], the origin could be local thermal desorption due to the power deposited by the ELMs. In fact, the observed $\mathrm{D}_{\alpha}$-emission location on top of Tiles 0 and 1 coincides with the region where most D containing Beryllium co-deposits accumulate, as deduced from surface analysis studies $[37,38]$. If this is true, in order to generate the outgassing of trapped D, an increased power load must occur that overheats the surfaces at this region that should be correlated with the observed local increased $\mathrm{D}_{\alpha}$-emission. Moreover, if this correlation is found, it would be interesting to understand the cause of this local overheating, most probably linked to the magnetic divertor configuration. These points will be analysed first in this section for the plasma described above.

For the discharge \#86533 analysed above, unfortunately the MWIR thermography camera looking to the inner divertor was not operative. We therefore studied the data from a NIR Protection Camera that looks tangentially with a similar view as the Fast Visible Camera. As disclosed in Section 2, these kind of cameras are only calibrated for very high temperatures $\mathrm{T}>800{ }^{\circ} \mathrm{C}$. Additionally, in many situations, other emission sources contaminate the thermal radiation filtered at this range, such as plasma Bremsstrahlung and emission due to atomic Free-bound transitions from recombining processes. This contamination can be even dominant especially in cool high-density plasma conditions as will be shown. Figure 5a shows a video clip of this NIR camera during the H-mode phase of plasma \#86533 (click on the frame to reproduce the video). The light intensity is in arbitrary units, i.e. without calibration. The transition from the $\mathrm{C}$ - to the $\mathrm{V}$ configuration takes place at about $t=6 \mathrm{~s}$ of the clip. The change in the emission pattern is clear. During the C-configuration, light at the inner divertor comes only from a narrow region corresponding to the SP line. When passing to the V-configuration, this emission cloud expands and becomes stronger, while brilliant emission regions appear on top of Tile 1. The origin of the toroidal emission cloud is volume plasma 
emission characteristic of a dense and cool plasma, but the light from the bright discrete areas with sharp contours at Tile 1 is mainly surface thermal emission indicating local overheating (Hot Spots). In Figure 5b the upper pictures show two frames of the same video (in false colour scale), left in C- and right in Vconfiguration. A Region of Interest (ROI) has been selected on top of Tile 1 around one of these hot spots and the time evolution of the average intensity in this ROI is represented below. The time trace shows a clear increase when the configuration changes from Corner to Vertical indicating a local temperature raise.

Since due the light contamination a quantitative estimation of the surface temperature is not possible for this plasma with the NIR Protection camera, another similar plasma of the same experiment was selected for which data of the MWIR thermography camera were available. Discharge \#84641 has a very similar divertor configuration change as the one analysed before (\#86533), although with $3 \mathrm{MW}$ less NBI power, no ICRF power and also somewhat lower $\mathrm{B}_{\mathrm{T}}$ and $\mathrm{I}_{\mathrm{P}}$. Figure 6a shows a frame of the MWIR camera for this discharge and below is a zoom of the divertor. Two ROIs were selected at Tiles 0 and 1 . The time evolution of the maximum temperature of both ROIs is shown in figure $6 \mathrm{~b}$ during the H-mode, the arrows indicating the $\mathrm{C}$ - and V-configuration phases. A clear raise of the base-temperature, from about $200-250{ }^{0} \mathrm{C}$ to 300 $400{ }^{\circ} \mathrm{C}$ is visible at Tiles 0 and 1 when the configuration changes to V. Additionally strong temperature spikes appear at Tile 1 reaching values $>700{ }^{\circ} \mathrm{C}$. Only a few ELM-crashes (duration time is $\approx 1 \mathrm{~ms}$ ) are randomly captured by the camera because its long "dead time" without data. In other words: since the time between two consecutive frames is $65 \mathrm{~ms}$ but the sensor exposure time just $0.6 \mathrm{~ms}$ only a fraction of ELMcrash events, that appear every $25 \mathrm{~ms}$ approximately, are captured. It should be noted that higher temperatures should be expected for the discharge analysed before (\#86533), since the heating power was higher.

On the other hand, laboratory measurements by Thermal Desorption Spectroscopy (TDS) have shown that the outgassing of D trapped in Beryllium co-deposits happen at discrete characteristic temperatures, which begin with a strong desorption peak at about $300-350{ }^{\circ} \mathrm{C}[39,40]$. This values seam to be reached and even well surpassed during ELMs at Tiles 0 and 1 during the V-configuration phase. We can therefore say that, at least potentially, during this plasma phase D may be desorbed from the Beryllium co-deposits. It should be however remembered that, due to the possible error in the temperature estimation (see section 2), this point should be confirmed in future studies as discussed later.

Once it is confirmed that for certain high-power H-mode plasmas the threshold temperature for D outgassing is very probably reached and surpassed at Tiles 0 and 1, it would be interesting to understand the origin of this anomalous overheating. Initially, when looking to the magnetic configuration change of the analysed plasma (figure 1), it was surprising, that a relatively small change of the inner SP position relative to the distance to the upper inner divertor region could produce such an increased surface heating where the D emission is observed. Also the modification of the ELM characteristics when changing the configuration cannot explain the increased heat load. Neither the ELM frequency nor the ELM energyloss (depicted from the pedestal $\mathrm{T}_{\mathrm{e}}$ and $\mathrm{n}_{\mathrm{e}}$ decays as well from the WI emission peak intensities) varied significantly with the configuration change. The only possible reason found for this temperature raise is following: when turning to V-configuration, the Clearance (or Gap) to the upper inner divertor corner at Tile 1, defined as the distance from the material surface to the Separatrix, was significantly reduced due to a change of the outer SP position. This can be seen in figure 1, where the respective Clearances are 
marked with a blue arrow for the $\mathrm{V}$-configuration (about $6 \mathrm{~cm}$ ) and a red one for the C-configuration (about $12 \mathrm{~cm}$ ). Assuming a typical SOL power decay length $\lambda_{\mathrm{q}}=3 \mathrm{~mm}$ for high-power H-mode plasmas in JET at the outer midplane and taking into a account the magnetic flux expansion at the upper inner divertor, which is the same for both configurations, we get that the heat flux becomes amplified there by a factor of about 30 in the V-configuration with the reduced Clearance.

On the other hand, it is well-known that co-deposits reach much higher temperatures compared to the to the bulk material on which they are deposited due to their low thermal conductivity [41-44]. In fact, they appear as glowing "Hot Spots" and can be distinguished quite easily from the bulk material even with visible cameras. This effect should also facilitate an enhanced overheating of these surfaces.

\section{Other examples}

To confirm the link between Clearance at the upper inner divertor with the increased surface heating and the enhanced $\mathrm{D}_{\alpha}$-emission there, other plasmas have been analysed. Figure 7a shows the divertor configuration of \#92121, where the position of the outer SP was changed along the outer Horizontal target during the H-mode phase. Note that in this case the inner SP was laid still at the vertical Tile 3. While doing this, the Clearance to the corner of Tile 1 was reduced from 10 to $6 \mathrm{~cm}$ approximately. The gas puffing rate and the NBI power $(\mathrm{P}=14 \mathrm{MW}$ ) was maintained constant. Here the only significant global plasma variation after the configuration change to a reduced Clearance was a $10 \%$ increase in plasma density and also a rise of the ELM frequency from 60 to $80 \mathrm{~Hz}$. Concerning the thermal and D visible emission changes we see following. Figure $7 b$ shows two images that are an average of ten frames corresponding to about $180 \mathrm{~ms}$ from the NIR Protection camera for the same discharge (again no MWIR camera available), left before and right after the Clearance decrease. The raise of the thermal emission from Hot Spots clearly indicates an increased power load there when the Clearance is reduced. Below in figure $7 \mathrm{c}$ are the images of a $D_{\gamma}$-filtered camera averaged over $200 \mathrm{~ms}$ with the same view $\left(\mathrm{D}_{\alpha}\right.$ was not available). Despite the unchanged inner SP position the emission distribution clearly changes: before the Clearance reduction the dominant $\mathrm{D}$ emission at the inner divertor comes from the lower part of the vertical target near the inner SP position and after from the top of Tile 1. Figure 7d shows the time evolution of the innermost divertor Spectroscopy $\mathrm{D}_{\alpha}$-emission chord (channel number 1) looking on top of Tiles 0 and 1. An increase of the base level (inter-ELM) during the Clearance reduction by a factor of about 6 is observed. At the same time (not shown), the equivalent channels of BeII and WI raise just by a factor of 2, as also does the ion saturation current of the innermost divertor Langmuir Probe on Tile 1 (see figure 1). This indicates that the Clearance reduction induces in this region an increase of the ion flux by a factor of about 2 while that of the $D_{\alpha}$-emission is three times larger. Since a sensible increase of S/XB cannot be expected, this means that the neutral flux is strongly enhanced with respect to the incoming ion flux that points again to local thermal desorption there. Figure $7 \mathrm{e}$ shows the clear displacement of the $\mathrm{D}_{\alpha}$-emission peak to the top of Tile 1 (channel number 2) during the inter-ELM period as obtained from the upper spectroscopy lines of view in agreement with the $\mathrm{D}_{\gamma}$-filtered camera (figure $7 \mathrm{c}$ ).

Another similar example of Clearance reduction without inner SP position change but where the MWIR thermography camera was available is shown in figure 8a. The two equivalent discharges \#83177 and 
\#83491 had following parameters: $\mathrm{B}_{\mathrm{T}}=2.8 \mathrm{~T}, \mathrm{I}_{\mathrm{P}}=2.5 \mathrm{MA}, \mathrm{P}=16 \mathrm{MW}(\mathrm{NBI})$. They were already compared in another work to see the effect when changing the outer SP from the horizontal to the vertical target [45]. The average images of the IR thermography video over 1 second (about 15 frames) of both plasmas during the H-mode of both discharges are shown to the right. For the plasma with reduced Clearance the temperature at Tiles 1 and 0 is of about of $300-350{ }^{\circ} \mathrm{C}$, much higher than the other one that just reaches about $200{ }^{\circ} \mathrm{C}$. Figure $8 \mathrm{~b}$ shows the inter-ELM $\mathrm{D}_{\alpha}$-emission distribution at the inner divertor $(1$ is innermost channel) for both discharges. The strong increase of the intensity for the reduced Clearance plasma from Tile 1 is again clearly visible.

The last case study compares three plasmas with following parameters: $\mathrm{B}_{\mathrm{T}}=2.2 \mathrm{~T}, \mathrm{I}_{\mathrm{P}}=2 \mathrm{MA}, \mathrm{P}=13.5$ MW (NBI) + 1.5 MW (ICRF). As shown in figure 9a, two of them had both SPs at the divertor although different puffing levels. In the third one the inner SP was moved to the vertical target. Note that the interesting point here is that the Clearance to the upper inner divertor corner is not varied, only the inner SP position. These plasmas were already analysed to study the influence of divertor neutral pumping optimisation and gas injection rate on plasma edge properties and global plasma confinement at high triangularity [10]. The best confinement $\left(\mathrm{H}_{98 \mathrm{Y}} \approx 1\right)$ was achieved for the lowest puffing level (lowest pedestal density) and both SPs at the divertor corners (\#89341). Stable plasmas with the inner SP at the vertical target with such low gas injection rates where not possible to maintain due to the high tungsten influx. This was only possible in discharge \#89340 when the gas level was duplicated, that had the same level as \#89334. In both plasmas the confinement degraded in by about $20 \%$ independently of the divertor configuration. Their pedestal densities were similar, although the ELM showed quite different characteristics [10], being much longer as in a quiscient H-mode for \#89340. Making a similar analysis for this set of plasmas as done above we observe following. The NIR protection camera showed that for increasing puffing levels, at the inner divertor an increasingly strong volume emission evolves, indication of the characteristic cool-high density recombining SOL plasma, this happening at a similar degree in the C- (\#89334) and the V-configuration (\#89340). However, no "Hot spots" are visible in none. The MWIR camera shows similar temperatures at Tiles 0 and 1 for both configurations. Additionally, as shown in figure $9 \mathrm{~b}$, the inter-ELM $\mathrm{D}_{\alpha}$-emission distribution at the inner divertor is very similar for the three plasmas. The one with the lowest puffing is by a factor of about 3 lower than the other two, which have similar intensities despite the different divertor configurations. There is no enhanced $\mathrm{D}_{\alpha}$-emission at the upper inner divertor for the plasma in the V-configuration here. Therefore it can be said that, moving the inner SP position from the divertor corner a few centimetres up on the vertical target, without Clearance modification, does not sensibly modify the neutral flux distribution.

\section{Using plasma configuration to induce fuel desorption from the upper inner divertor}

It is well known that the plasma Hydrogen isotope is not only composed of that injected by puffing to the plasma or by NBI, but also of the ones trapped in the reactor surface walls and re-enter into the plasma during recycling. This is called "Wall isotope exchange". This process is used intentionally as a Wall-conditioning technique to clean-up the reactor vessel. It has been already used in TFTR and JET to retrieve the trapped $\mathrm{T}$ from the walls after D-T experiments with repetitive low power plasmas [46-49]. 
Separatrix scanning with high power plasmas has been also proposed elsewhere [50]. Wall isotope exchange has been also extensively studied using ICRF discharges with only toroidal magnetic fields to apply it to future superconducting reactors such as ITER [51-53]. A review on the different Wallconditioning techniques proposed for ITER can be found in [54].

More recently, dedicated experiments of isotope exchange in JET with ITER-like wall using Ohmic and low-power L-mode plasmas showed a very weak access to the long-term retention reservoir in the codeposited Beryllium layers where most of the fuel becomes trapped [55]. Therefore alternative techniques to clean up the retained $\mathrm{T}$ in JET would be desirable. As already mentioned, it is known that fuel trapped in Beryllium co-deposits in the divertor can be removed by heating the surface to temperatures $>300$ $350{ }^{\circ} \mathrm{C}[37,38]$. Unfortunately, such high temperatures can be reached by baking of the vacuum vessel in only marginally. Therefore, specific Wall-conditioning techniques are proposed to prepare the next JET T campaigns with the ITER-like wall [56]. One of the proposed techniques is linked to the subject-matter of the present work, since the local outgassing from the Be co-deposits at the upper inner divertor is intentionally induced by plasmas with magnetic configurations that focalise the ion and heat fluxes there. A preliminary analysis is shown in this section.

The discharge described here corresponds to a plasma from the beginning of a $\mathrm{D}$ campaign just after an $\mathrm{H}$ one. Figure 10a shows the magnetic configuration of the high-power plasma \#91961 with $\mathrm{B}_{\mathrm{T}}=3 \mathrm{~T}, \mathrm{I}_{\mathrm{P}}=$ 3.2 MA and $\mathrm{P}=24 \mathrm{MW}(\mathrm{NBI})$. It was used to study $\mathrm{W}$ melting at Tile 5 by directing the outer SP there. The inner SP was raised at the upper corner of Tile 1 and the Clearance there was quite small $(6 \mathrm{~cm})$. Figure 10b up corresponds to a frame of the MWIR thermography camera during the NBI-phase and where two ROIs are defined corresponding to Tiles 0 and 1 . Below, the corresponding temperature evolutions are shown. The ROI corresponding to Tile 1 overpasses the camera settings saturation value with $\mathrm{T}>1100{ }^{\circ} \mathrm{C}$ (note that half the value is shown) and the one of Tile 0 reaches $600{ }^{\circ} \mathrm{C}$. For these very high temperatures a strong outgassing at the upper inner divertor region has to be expected.

Figure 11 shows following time traces for this discharge: the NBI power $\mathrm{P}$, the $\mathrm{D}$ puffing rate $\mathrm{Q}_{0}$, the pressure measured in the lower sub-divertor duct $p$, the vertical coordinate of the inner SP $Z_{S P}$ and the innermost $\mathrm{D}_{\alpha \square} \square$ chord $\mathrm{L}_{\mathrm{D} \alpha \text { in }}$. $\mathrm{Q}_{0}$ has at the beginning a pulse (marked with a circle) to achieve the desired plasma density for the H-mode and later stays constant at a moderate value. The corresponding increase of the pressure $p$ to this puffing pulse is marked with a first red arrow. There is a time delay of $p$ with respect to the puffing pulse due to the conductance of the tokamak vacuum vessel to the manometer location, that is quite far. The inner $\mathrm{SP}$ is raised at $\mathrm{t} \geq 12 \mathrm{~s}$, when $\mathrm{Z}_{\mathrm{SP}}$ increases as marked with a second circle. Despite the constant puffing level, the neutral pressure shows a second increase with a similar time delay as before. Also at this moment, the surface temperatures of Tiles 0 and 1 sharply raise (figure 10b). At the same time the innermost $D_{\alpha \square} \square$ flux strongly increases and even saturates $\left(L^{D \alpha}>4 \times 10^{17} \mathrm{ph} \mathrm{s}^{-1} \mathrm{~cm}^{-2} \mathrm{sr}^{-1}\right)$, this indicating a strong recycling/outgassing source at the upper inner divertor as we expected before.

The here analysed discharge was one of the first high power plasmas of a $\mathrm{D}$ campaign just after a long $\mathrm{H}$ campaign and one could expect that $\mathrm{H}$ isotope was accumulated in the Beryllium co-deposits of Tiles 0 and 1. Therefore, if local heating and fuel outgassing was achieved by this D plasma, than some accumulated $\mathrm{H}$ isotope should have been desorbed. The lowest frame of figure 11 shows the time trace of the $\mathrm{H}$ isotope concentration $(H /(H+D))$ of the plasma as measured by an optical Penning gauge in the sub-divertor. There 
is a clear increase of this signal, that reached up to about $10 \%$ during the phase when Tiles 0 and 1 were heated due to the inner SP raise. Note that before this, the H-concentration is $\leq 3 \%$, which is below the detection limit. This is a clear indication that the plasma configuration with the raised inner SP was effective in desorbing trapped $\mathrm{H}$ at the upper inner Divertor region.

Future studies are planed before the next $\mathrm{T}$ campaign to try to confirm the potential use of this local plasma heating and outgassing effect at the upper inner divertor as a Wall-conditioning technique for JET [50]. For the next T-T experiments, it is desired to remove all possible trapped D from the walls of previous campaigns, to minimise $\mathrm{D}-\mathrm{T}$ fusion reactions. A H-campaign has been foreseen before the $\mathrm{T}$ one, to exchange as much as possible $\mathrm{H}$ by the previously trapped D. In this phase, the use of raised inner SP configurations to induce heating of Tiles 0 and 1 will be studied in more detail. If useful, the same technique could be later applied to recover surface retained $\mathrm{T}$ at this region.

\section{Discussion and Summary}

In the present work we have studied some aspects concerning the impact of the divertor configuration on recycling neutral fluxes for JET ITER-like wall high power H-mode plasmas. We analyse in detail the previously observed anomalously strong $\mathrm{D}_{\alpha}$-emission [22] that appears at the upper inner divertor for certain magnetic topologies. For these plasmas, a systematic correlation has been found of this enhanced emission with a local surface temperature raise there. Since this region is where the thickest fuel containing Be co-deposits accumulate, we believe that surface overheating of these are the origin of the outgassing. In fact, the measured surface temperatures locally exceed the typical thermal D release from this kind of layers $\left(\mathrm{T}>350{ }^{0} \mathrm{C}\right)$. The $\mathrm{D}$ emission happens just after the ELM-crash for a period of typically 5 milliseconds. Its flux was quantified and dominates over puffing and the rest of recycling during this period of the ELM-cycle.

We propose that the origin of the local increased surface temperature for these plasma configurations is the increased heat flux due to the reduced distance from inner upper divertor corner to the Separatrix (Clearance). Additionally, it is well-known that co-deposits reach more easily higher temperatures compared to the to the bulk material on which they are deposited due to their low thermal conductivity. It must be however acknowledged, that there is a considerable uncertainty in the present absolute surface temperature measurements. This is because the co-deposits on top of the $\mathrm{W}$-coated carbon Tiles 0 and 1 most probably alters the estimated thermal surface emissivity, which is a fundamental parameter to deduce the temperature. Future work is on going to experimentally measure the emissivity more accurately there.

On the other hand it should be noted that the Clearance reduction at the upper inner divertor may have additional effects apart from those described in the present work. It seams obvious to believe that the coupling between the divertor SOL and the confined pedestal plasma region will be facilitated. For example, the penetration probability inside the Separatrix of the neutrals (fuelling efficiency) coming from this region will be increased due to the shorter path. Also the SOL radiation layer, which generally dominates at the inner divertor region close to the X-point, has a shorter distance to approach and even penetrate into the confined pedestal plasma region (perhaps inducing its cooling). Summarising, the Clearance reduction at the upper inner divertor may, additionally to the effects described in the present 
work, also affect the coupling of the inner divertor SOL plasma with the confined pedestal region that should be taken into account when analysing the effect of the magnetic topology.

Finally, we have studied high-power plasmas with raised inner SP at the upper inner divertor corner to intentionally induce previously trapped D by thermal outgassing. The intention is to use this as a fuel recovery technique at regions where Be co-deposits accumulate. By focalising the plasma ion and heat flux there, very high temperatures can be achieved $\left(\mathrm{T}>1000^{\circ} \mathrm{C}\right)$ and as expected, strong $\mathrm{D}$ desorption from this region was observed. The shown preliminary analysis indicates that these plasmas can be used for Wall isotope exchange and $\mathrm{T}$ recovery specifically in this reactor vessel region, complementarily to other WallConditioning techniques [56]. More specific experiments are planed in the next future to continue this study and optimise the plasma parameters (power, magnetic shape, density, etc) for maximum conditioning efficiency.

\section{Acknowledgement}

This work has been carried out within the framework of the EURO fusion Consortium and has received funding from the Euratom research and training programme 2014-2018 under grant agreement No 633053. The views and opinions expressed herein do not necessarily reflect those of the European Commission.

\section{References}

[1] S Brezinsek et al. 2013 Nucl. Fusion 53083023

[2] G F Matthews et al. 2014 Phys. Scr. T159 014015

[3] F Romanelli on behalf of JET Contributors 2015 Nucl. Fusion 55104001

[4] X Litaudon et al. 2017 Nucl. Fusion 57102001

[5] C Giroud et al. 2013 Nucl. Fusion 53113025

[6] E de la Luna et al. $201425^{\text {th }}$ IAEA FEC, Saint Petersburg, Russia

[7] E Joffrin et al. $201425^{\text {th }}$ IAEA FEC, Saint Petersburg, Russia

[8] E Solano et al. 2014 Proc. 41 st EPS Conference on Plasma Physics, Berlin, Germany

[9] P Tamain et al. 2015 J. Nucl. Mat. 463450

[10] E de la Luna et al. 2016 IAEA FEC 2016

[11] E Joffrin et al. 2017 Nucl. Fusion 57086025

[12] S Kaye et al. 1984 J. Nucl. Mater. 121115

[13] L.D. Horton et al. 1999 Nucl. Fusion 391

[14] A Loarte 2001 Plasma Phys. Control. Fusion 43 R183

[15] A S Kukushkin et al. 2005 Nucl. Fusion 45608

[16] S Brezinsek et al. 2015 J. Nucl. Mat. 46311

[17] M Groth et al. 2013 Nucl. Fusion 53093016

[18] A Järvinen et al. 2013 Proc. 40th EPS Conf. on Plasma Physics, Espoo, Finland.

[19] S Wiesen et al. 2016 Contrib. Plasma Phys. 56754

[20] D M Harting et al. 2015 J. Nucl. Mat. 463493 
[21] S Brenzisek et al. 2016 Phys. Scripta T167 014076

[22] S Wiesen et al 2017 Nucl. Fusion 57066024

[23] C Guillemaut et al. 2018 Nucl. Fusion 58066006

[24] E Marenkov et al. 2012 Phys. Plasmas 19092501

[25] A Yu Pigarov et al. 2014 Phys. Plasmas 21062514

[26] E de la Cal and the TJ-II Team 2015 Plasma Phys. Control. Fusion 57075001

[27] K Schmid 2016 Phys. Scripta T167 014025

[28] V S Neverov et al. 2019 Nucl. Fusion 59046011

[29] L Horton et al. 2016 Fusion Eng. Design 109-111 925

[30] E de la Cal et al. 2012 Proc. 39th EPS Conf. on Plasma Physics (Stockholm, Sweden)

[31] A Huber et al. 2012 Rev. Sci. Instr. 83 10D511

[32] E Gauthier et al. 2007 Fusion Eng. Design 821335

[33] I Balboa et al. 2016 Rev. Sci. Instr. 87 11D419

[34] G Arnoux et al. 2012 Rev. Sci. Instr. 83 10D727

[35] A Huber et al. 2017 Phys. Scripta T170 014027

[36] V Huber et al. 2017 Fusion Eng. Design 123979

[37] M Mayer et al. 2016 Phys. Scr. T167 014051

[38] A Widdowson et al. 2017 Nucl. Fusion 57086045

[39] D Alegre et al. 2017 Physica Scripta T170 014028

[40] G De Temmerman et al. 2017 Nucl. Mat. Energy 12267

[41] P H LaMarche et al. 1986 J. Vac. Sci. Tech. A 41198

[42] E Gauthier et al. 2005 J. Nucl. Mat. 337-339 960

[43] J Gaspar et al. 2016 Int. J. Thermal Sci. 104292

[44] R Mitteau et al. 2006 Nucl. Fusion 46 S49

[45] M J Leyland et al. 2015 Nucl. Fusion 55013019

[46] L D Horton et al J. Nucl. Mat. 196-198 139-142

[47] P Andrew et al.1992 J. Nucl. Mater. 196-198 143

[48] C H Skinner et al. 1997 J. Nucl. Mat. 241-243 214

[49] P Andrew et al. 1999 Fusion Eng. Design 47233

[50] S Higashijima et al.1999 J. Plasma Fusion Res. 751297 (in Japanese)

[51] E de la Cal and E Gauthier 1997 Plasma Phys. Control. Fusion 391083

[52] D Douai et al. 2011 J. Nucl. Mat. 415 S1021

[53] T Wauters et al. 2011 J. Nucl. Mat. 415 S1033

[54] M Shimada et al. 2011 J. Nucl. Mat. 415 S1013

[55] T Loarer et al. 2015 Nucl. Fusion 55043021

[56] I Borodkina et al. 2018 Proc. 45th EPS Conf. on Plasma Physics O2.106, Prague, 2018

[57] G M McCracken et al. 1998 Nucl. Fusion 38619

\section{Appendix}

The use of equation (1) to estimate the $\mathrm{D}$ neutral flux $\mathrm{F}_{0}$ through the $\mathrm{D}_{\alpha}$-radiance $\mathrm{L}_{\mathrm{D} \alpha}$ can be only used if the plasma is in Ionizing conditions, i.e. recombination can be neglected. This has been checked to be the case here in two ways: 
1. The ion flux of the upper Langmuir probe (see figure 1) estimated from the ion saturation has a similar value (within a factor of two) than the neutral flux obtained locally from $\mathrm{D}_{\alpha}$-channel number 3 , that looks very near to this probe. When the measured ion and neutral fluxes are similar, it means that recycling dominates over recombination.

2. It is known that in low temperature, high density recombining plasmas, the ratio of the Balmer lines $\mathrm{D}_{\gamma} / \mathrm{D}_{\alpha \square}$ increases from about 0.02 in Ionizing conditions to about 0.1 in Recombining conditions [57]. 


\section{Figure Captions}

Figure 1: Divertor magnetic configurations of plasma \#86533 showing the Corner (C) and Vertical (V) configurations. Shown are also some inner $\mathrm{D}_{\alpha}$-channels and Langmuir probes (LP).

Figure 2: Time traces of the NBI power $P$, puffing rate $Q_{0}$, plasma average density $\left\langle n_{e}>\right.$, pedestal electron temperature $\mathrm{T}_{\mathrm{e}}$, the $\mathrm{H}_{98 \mathrm{Y}}$ confinement factor and the integrated inner and outer divertor $\mathrm{D}_{\alpha}$-emission radiance $\mathrm{L}_{\mathrm{D} \alpha}$. The arrows at the horizontal axis mark the $\mathrm{C}$ - and $\mathrm{V}$-configuration phases.

Figure 3: a) Two frames of the divertor taken with the visible Fast camera during the V-configuration of \#86533: left before the ELM and right after the ELM and below the CAD view of the camera to the divertor (see text). b) Corresponding video-clips of the Corner- (up) and the Vertical-configuration (down), (click on the frame to reproduce the video).

Figure 4: a) Inter-ELM level evolution of the innermost $\mathrm{D}_{\alpha}$-channel radiance $\mathrm{L}^{\mathrm{D} \alpha}$ looking to the corner between Tiles 0 and 1.b) Time traces of $\mathrm{D}_{\alpha^{-}}$radiance of channels 1, 3 and 5 (see Figure 1) and total inner divertor WI radiance during C- (up) and V-configuration (down).

Figure 5: a) Video clip of NIR camera during the H-mode phase of \#86533 (click on the frame to reproduce the video). b) Upper pictures show two frames of the same video (in false colour scale), left in $\mathrm{C}$ - and right in $\mathrm{V}$-configuration and below the time evolution of the average intensity of the selected ROI.

Figure 6: a) Wide-angle view of the MWIR camera for plasma \#84641 and below a Zoom of the divertor where two ROIs at Tiles 0 and 1 are shown. b) Time evolution of the maximum temperature of the selected ROIs during the $\mathrm{H}$-mode, the arrows indicating the $\mathrm{C}$ - and $\mathrm{V}$-configuration phases.

Figure 7: a) Divertor configuration of \#92121 and b) two averaged images of the NIR camera and c) $D_{\gamma^{-}}$ filtered camera. The left and right images correspond respectively to the larger and shorter Clearance phases. d) Time evolution of the innermost divertor Spectroscopy $\mathrm{D}_{\alpha}$-emission chord looking to Tiles 0 and 1. e) $D_{\alpha}$-emission profiles as a function of channel number and major radius $R$.

Figure 8: a) Magnetic configurations of plasmas \#83177 and \# 83491 and to the right, two frames of each of the MWIR. b) Inter-ELM $\mathrm{D}_{\alpha}$-emission profiles at the inner divertor of both plasmas.

Figure 9: a) Magnetic configurations of plasmas \#89341, \#89334 and \#89340 and b) inter-ELM $\mathrm{D}_{\alpha^{-}}$ emission distribution at the inner divertor. 
$1 \quad$ Figure 10:a) Magnetic configuration of plasma \#91961 and b) frame of the MWIR thermography camera during the NBI-phase where two ROIs are defined corresponding to Tiles 0 and 1 and below, their corresponding temperature evolutions (note that the half value is shown for Tile 1).

Figure 11: Time traces of plasma \#91961: NBI power $P$, puffing flux $Q_{0}$, pressure measured in the subdivertor $\mathrm{p}$, vertical coordinate of the inner $\mathrm{SP} \mathrm{Z}_{\mathrm{SP}}$, the innermost $\mathrm{D}_{\alpha \square} \square$ chord $\mathrm{L}_{\mathrm{D} \alpha \text { in }}$ and Hydrogen concentration $\mathrm{H} /(\mathrm{H}+\mathrm{D})$. 